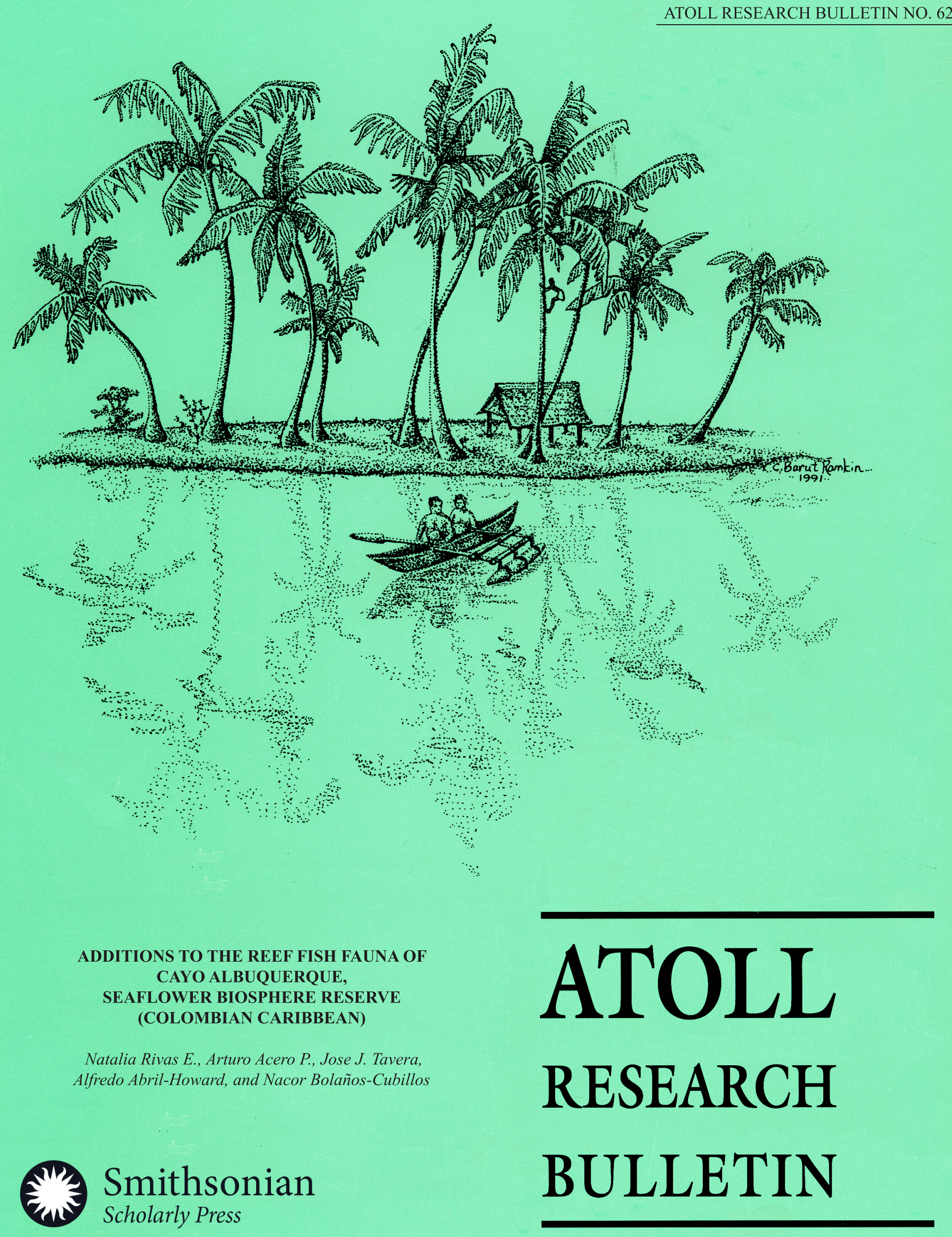





\section{ADDITIONS TO THE REEF FISH FAUNA OF CAYO ALBUQUERQUE, SEAFLOWER BIOSPHERE RESERVE (COLOMBIAN CARIBBEAN)}

Natalia Rivas E., Arturo Acero P., Jose J. Tavera, Alfredo Abril-Howard, and Nacor Bolaños-Cubillos

Atoll Research Bulletin No. 627 • 9 December 2020

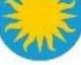

Smithsonian

Scholarly Press

Washington, D.C. 
All statements made in papers published in the Atoll Research Bulletin are the sole responsibility of the authors and do not necessarily represent the views of the Smithsonian Institution or of the editors of the bulletin. Articles submitted for publication in the Atoll Research Bulletin should be original papers and must be made available by authors for open access publication under a CC BY-NC license. Manuscripts should be consistent with the "Author Formatting Guidelines for Publication in the Atoll Research Bulletin." All submissions to the bulletin are peer reviewed and, after revision, are evaluated prior to acceptance and publication through the publisher's open access portal (https://smithsonian.figshare.com/ScholarlyPress).

Published by SMITHSONIAN INSTITUTION SCHOLARLY PRESS

P.O. Box 37012, MRC 957

Washington, D.C. 20013-7012

https://scholarlypress.si.edu/

Copyright (C) 2020 Natalia Rivas E., Arturo Acero P., Jose J. Tavera, Alfredo Abril-Howard, and Nacor Bolaños-Cubillos

ISSN: 0077-5630 (online)

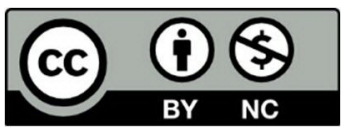

This work is licensed under a Creative Commons Attribution-NonCommercial 4.0 International License. 


\section{CONTENTS}

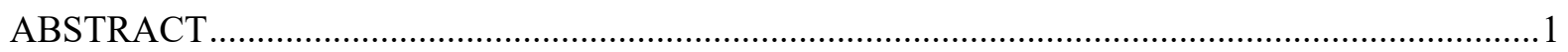

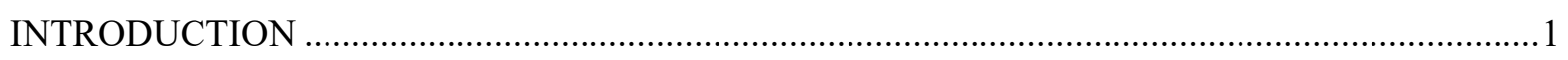

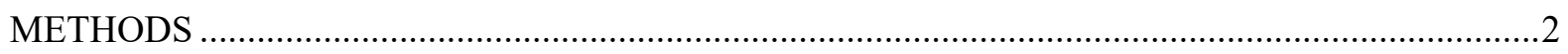

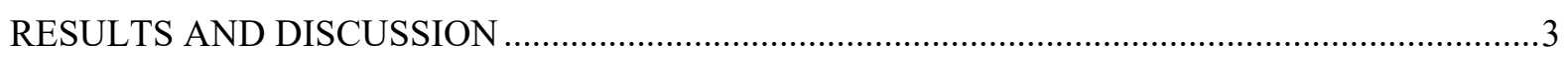

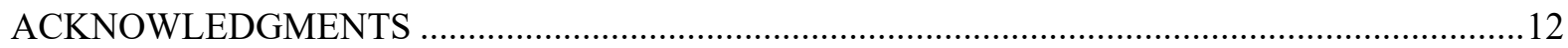

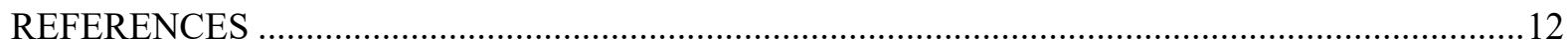





\title{
ADDITIONS TO THE REEF FISH FAUNA OF CAYO ALBUQUERQUE, SEAFLOWER BIOSPHERE RESERVE (COLOMBIAN CARIBBEAN)
}

\author{
NATALIA RIVAS E. ${ }^{1}$, ARTURO ACERO P. ${ }^{1}$, JOSE J. TAVERA ${ }^{2}$, \\ ALFREDO ABRIL-HOWARD ${ }^{1}$, and NACOR BOLAÑOS-CUBILLOS ${ }^{3}$
}

\begin{abstract}
This publication presents the list of fish fauna gathered during a scientific expedition to Cayo Albuquerque $\left(12^{\circ} 07^{\prime} \mathrm{N}, 81^{\circ} 52^{\prime} 2^{\prime \prime}\right.$ W) Colombia, the southernmost cay of the Archipelago of San Andrés, Providencia and Santa Catalina, which includes the Seaflower Biosphere Reserve. Data was collected through visual censuses in coral reef formations and seagrass beds, between September 23 and October 3, 2018. A total of 191 species were registered, distributed in 50 families, with Labridae (24) and Serranidae (22) showing the highest species richness. Twenty-five species are new reports for Albuquerque.
\end{abstract}

\section{INTRODUCTION}

The Archipelago of San Andrés, Providencia and Santa Catalina in the western Caribbean is composed of three main islands and a large number of small islands, cays, atolls, and shoals that stretch from northeast to southwest over $\sim 180,000 \mathrm{~km} 2$ (Abril-Howard et al., 2012; Gamboa et al., 2012) (Figure 1). In recognition of the archipelago's size, location, fishing resources, species diversity, ecosystem richness, and other attributes, UNESCO in 2000 designated the archipelago as the Seaflower Biosphere Reserve (Taylor et al., 2012).

The Seaflower Biosphere Reserve encompasses significant Caribbean coral reef complexes and 77\% of the coral reef formations of Colombia (Abril-Howard et al., 2012). Since 2015, Colombian research institutions have participated in annual scientific expeditions to remote areas of the Reserve, to increase knowledge of their ecosystems and species richness. This paper presents the list of fish fauna inventoried during the 2018 expedition to the southern limit of the reserve, Cayo Albuquerque (Southwest Cay; Figure $1)$.

\footnotetext{
${ }^{1}$ Instituto de Estudios en Ciencias del Mar, CECIMAR, Universidad Nacional de Colombia, Playa Salguero, Santa Marta, Colombia. narivase@unal.edu.co

${ }^{2}$ Laboratorio de Ictiología, Grupo de Investigación SEyBA, Departamento de Biología, Universidad del Valle, Cali, Colombia

${ }^{3}$ Corporación para el Desarrollo Sostenible del Archipiélago de San Andrés, Providencia y Santa CatalinaCORALINA, San Andrés, Colombia.
} 


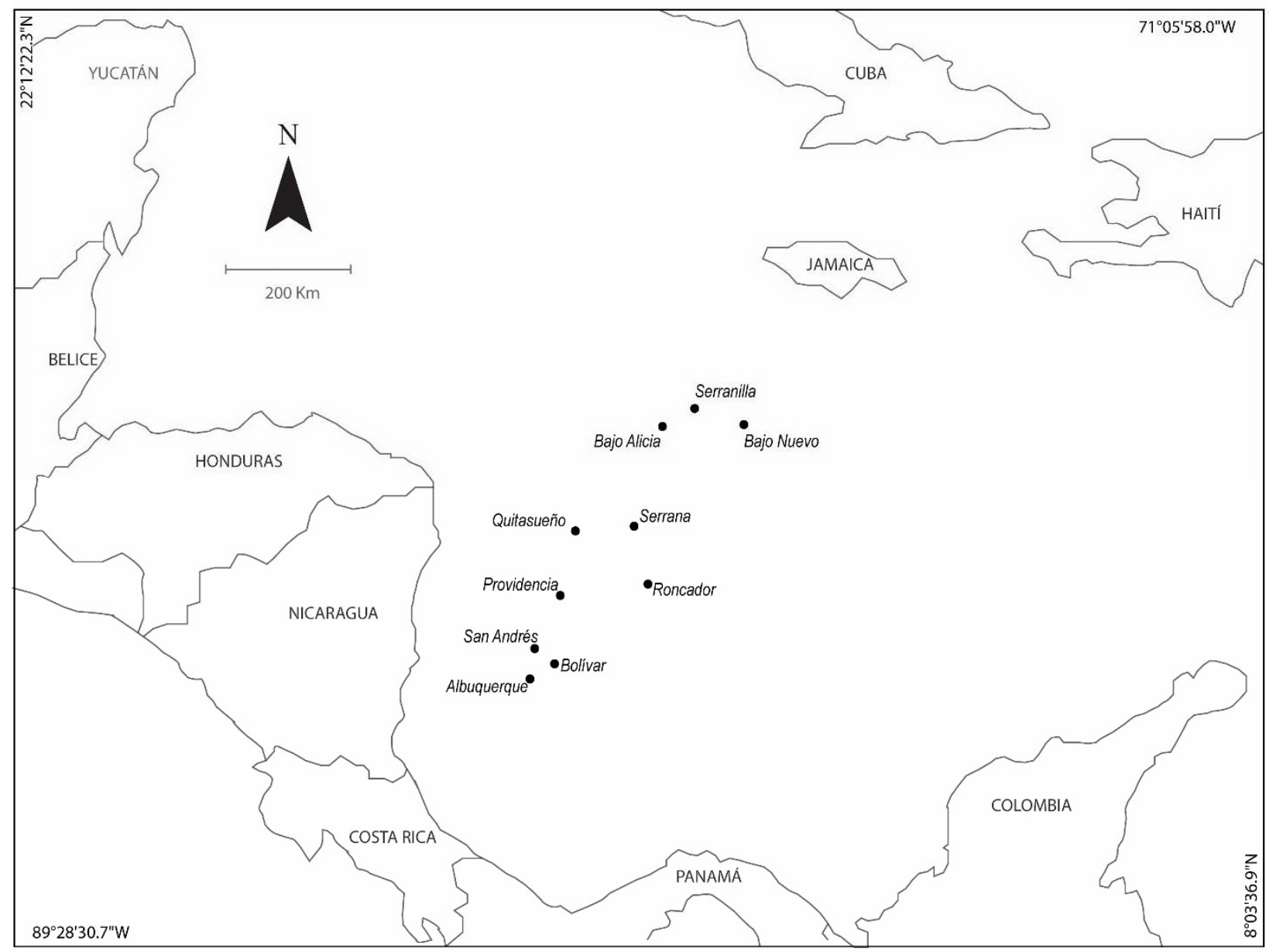

Figure 1. Archipelago of San Andres, Providencia and Santa Catalina.

\section{METHODS}

Data were gathered during the Seaflower expedition to Cayo Albuquerque $\left(12^{\circ} 07^{\prime} \mathrm{N}, 81^{\circ} 52^{\prime} 2^{\prime \prime} \mathrm{W}\right)$, from September 23 to October 3, 2018. In each of 35 stations (Figure 2) of coral reefs and seagrass beds (2 to $30 \mathrm{~m}$ deep), 30 minute underwater visual surveys were carried out using SCUBA and free-diving techniques. In addition, species recorded (with video or still photography) by the project "Diversity and relative abundance of elasmobranchs in Cayo Albuquerque" (Colombia Azul Foundation, NOVA Southern University, and Florida International University) and by other expedition scientists were included.

The main source of census comparisons were reef data from (i) Bolaños-Cubillos et al. (2015), to determine which reports were new to the cays, and (ii) Acero et al. (2019), which summarizes the fish biodiversity data collected during former Seaflower Expeditions (2015-2017) at the northern portion of the Reserve, including the islands of Roncador, Serrana, and Serranilla. 


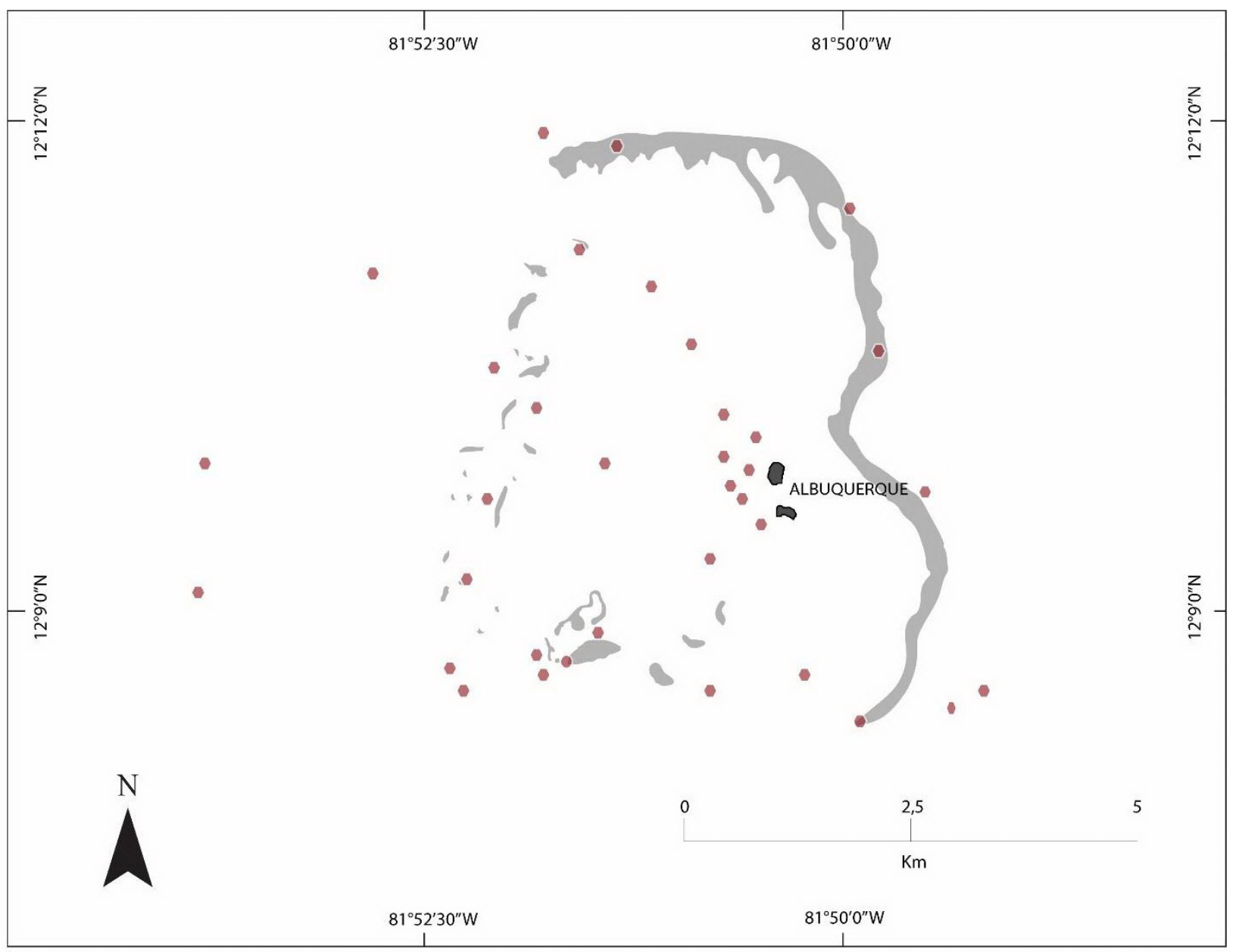

Figure 2. Sampling stations (red circles) and coral reef formations (light gray areas) around Cayo Albuquerque. Adapted from Taylor et al. (2012).

\section{RESULTS AND DISUSSION}

A total of 191 fish species from 50 families were observed at the stations surveyed around Albuquerque. Previous reports of the ichthyofauna of the cays (Fowler, 1944; Birdsong and Emery, 1967; Victoria and Gómez, 1984; and Gómez and Victoria, 1986) were included in the list compiled by Mejía et al. (1998), which reports a total of 119 species. After a rigorous compilation of those works and additional sampling, Bolaños-Cubillos et al. (2015) presented a list of 336 species recognized for the south section of the reserve (Bolívar and Albuquerque; Figure 1).

This work adds 25 new reports to the known species of Cayo Albuquerque (Table 1). Labridae is the family that has the highest species richness (24), including four species not previously detected in the cays (Cryptotomus roseus, Halichoeres pictus, H. poeyi, and Xyrichtys martinicensis). Serranidae is represented by 22 species in total, including three new reports for the study area (Hypoplectrus maculiferus, $H$. randallorum, and Serranus baldwini).

Amongst the islands sampled in previous Seaflower Expeditions, Albuquerque exhibited the highest fish species richness (191), compared to 140 species recognized for Roncador in 2015, 155 for Serrana in 2016, and 166 for Serranilla in 2017 (Acero et al., 2019). The fish biodiversity found in Albuquerque during 
the short visual census includes more than $12 \%$ of the total number of species recognized for the Greater Caribbean in depths shallower than 100 m (Robertson et al., 2019).

The information gathered in Albuquerque is consistent with data from the northern islands (Acero et al., 2019), specifically the relatively low abundance and/or absence of larger, generally high commercial value species such as Seriola dumerili, Calamus bajonado, Scarus coeruleus, Epinephelus striatus, Mycteroperca bonaci, and M. venenosa, among others. This is probably related to fishing pressure inside the reserve (Chasqui et al., 2017) and indicates the urgent need to continue monitoring and evaluating species richness and populations status of these species.

Table 1. Fish biodiversity found during a short visual census in Cayo Albuquerque, Seaflower Biosphere Reserve (Colombian Caribbean). The list is organized phylogenetically; orders and families of cartilaginous fishes followed Nelson et al. (2016) and bony fishes Betancur-R et al. (2017). * indicates a new report for Cayo Albuquerque. ${ }^{*} D$ indicates a contribution by Diego Cardeñosa, Colombia Azul Foundation. Families with uncertain ordinal status are listed as incertae sedis within the Series Carangaria, Ovalentaria, and Eupercaria (Betancur-R et al., 2017).

\begin{tabular}{|c|c|c|}
\hline ORDER & FAMILY & SPECIES \\
\hline Orectolobiformes & Ginglymostomatidae & Ginglymostoma cirratum (Bonnaterre, 1788) \\
\hline \multirow{3}{*}{ Carcharhiniformes } & \multirow{2}{*}{ Carcharhinidae } & Carcharhinus perezii (Poey, 1876) \\
\hline & & Rhizoprionodon terraenovae (Richardson, 1836) *D \\
\hline & Sphyrnidae & Sphyrna mokarran (Rüppell, 1837) *D \\
\hline \multirow{2}{*}{ Myliobatiformes } & Dasyatidae & Hypanus americanus (Hildebrand \& Schroeder, 1928) \\
\hline & Myliobatidae & Aetobatus narinari (Euphrasen, 1790) \\
\hline \multirow{9}{*}{ Anguilliformes } & \multirow{5}{*}{ Muraenidae } & Echidna catenata (Bloch, 1795) * \\
\hline & & Gymnothorax funebris Ranzani, 1839 \\
\hline & & Gymnothorax miliaris (Kaup, 1856) \\
\hline & & Gymnothorax moringa (Cuvier, 1829) \\
\hline & & Gymnothorax vicinus (Castelnau, 1855) \\
\hline & Congridae & Heteroconger longissimus Günther, 1870 \\
\hline & \multirow{3}{*}{ Ophichthidae } & Ahlia egmontis (Jordan, 1884) \\
\hline & & Myrichthys breviceps (Richardson, 1848) \\
\hline & & Ophichthus ophis (Linnaeus, 1758) * \\
\hline
\end{tabular}


Table 1. Continued

\begin{tabular}{|c|c|c|}
\hline \multirow{3}{*}{ Aulopiformes } & \multirow{3}{*}{ Synodontidae } & Synodus intermedius (Spix \& Agassiz, 1829) \\
\hline & & Synodus saurus (Linnaeus, 1758) * \\
\hline & & Synodus synodus (Linnaeus, 1758) \\
\hline \multirow{5}{*}{ Holocentriformes } & \multirow{5}{*}{ Holocentridae } & Holocentrus adscensionis (Osbeck, 1765) \\
\hline & & Holocentrus rufus (Walbaum, 1792) \\
\hline & & Myripristis jacobus Cuvier, 1829 \\
\hline & & Neoniphon marianus (Cuvier, 1829) \\
\hline & & Neoniphon vexillarium (Poey, 1860) \\
\hline \multirow{4}{*}{ Syngnathiformes } & \multirow{2}{*}{ Mullidae } & Mulloidichthys martinicus (Cuvier, 1829) \\
\hline & & Pseudupeneus maculatus (Bloch, 1793) \\
\hline & Callionymidae & Callionymus bairdi (Jordan, 1888) \\
\hline & Aulostomidae & Aulostomus maculatus Valenciennes, 1841 \\
\hline \multirow{5}{*}{ Kurtiformes } & \multirow{5}{*}{ Apogonidae } & Apogon lachneri Böhlke, 1959 \\
\hline & & Apogon maculatus (Poey, 1860) \\
\hline & & Apogon pseudomaculatus Longley, 1932 * \\
\hline & & Apogon quadrisquamatus Longley, 1934 \\
\hline & & Apogon townsendi (Breder, 1927) \\
\hline \multirow{9}{*}{ Gobiiformes } & \multirow{7}{*}{ Gobiidae } & Coryphopterus dicrus Böhlke \& Robins, 1960 \\
\hline & & Coryphopterus eidolon Böhlke \& Robins, 1960 \\
\hline & & Coryphopterus personatus (Jordan \& Thompson, 1905) \\
\hline & & Coryphopterus tortugae (Jordan, 1904) * \\
\hline & & Elacatinus evelynae (Böhlke \& Robins, 1968) \\
\hline & & Elacatinus horsti (Metzelaar, 1922) \\
\hline & & Priolepis hipoliti (Metzelaar, 1922) \\
\hline & \multirow{2}{*}{ Oxudercidae } & Ctenogobius saepepallens (Gilbert \& Randall, 1968) * \\
\hline & & Gnatholepis thompsoni Jordan, 1904 \\
\hline
\end{tabular}


Table 1. Continued

\begin{tabular}{|c|c|c|}
\hline ORDER & FAMILY & SPECIES \\
\hline \multirow{2}{*}{$\begin{array}{l}\text { incertae sedis- } \\
\text { Carangaria }\end{array}$} & \multirow{2}{*}{ Sphyraenidae } & Sphyraena barracuda (Edwards, 1771) \\
\hline & & Sphyraena picudilla Poey, 1860 *§ \\
\hline \multirow{12}{*}{ Carangiformes } & \multirow{2}{*}{ Echeneidae } & Echeneis naucrates Linnaeus, 1758 \\
\hline & & Remora remora (Linnaeus, 1758) * \\
\hline & \multirow{10}{*}{ Carangidae } & Caranx bartholomaei (Cuvier, 1833) \\
\hline & & Caranx crysos (Mitchill, 1815) \\
\hline & & Caranx hippos (Linnaeus, 1766) \\
\hline & & Caranx latus Agassiz, 1831 \\
\hline & & Caranx lugubris Poey, 1860 \\
\hline & & Caranx ruber (Bloch, 1793) \\
\hline & & Elagatis bipinnulata (Quoy \& Gaimard, 1825) \\
\hline & & Seriola rivoliana Valenciennes, 1833 \\
\hline & & Trachinotus falcatus (Linnaeus, 1758) \\
\hline & & Trachinotus goodei Jordan \& Evermann, 1896 \\
\hline Pleuronectiformes & Bothidae & Bothus lunatus (Linnaeus, 1758) \\
\hline \multirow{10}{*}{$\begin{array}{l}\text { incertae sedis- } \\
\text { Ovalentaria }\end{array}$} & \multirow{10}{*}{ Pomacentridae } & Abudefduf saxatilis (Linnaeus, 1758) \\
\hline & & Abudefduf taurus (Müller \& Troschel, 1848) \\
\hline & & Chromis cyanea (Poey, 1860) \\
\hline & & Chromis insolata (Cuvier, 1830) \\
\hline & & Chromis multilineata (Guichenot, 1853) \\
\hline & & Microspathodon chrysurus (Cuvier, 1830) \\
\hline & & Stegastes adustus (Troschel, 1865) \\
\hline & & Stegastes diencaeus (Jordan \& Rutter, 1897) \\
\hline & & Stegastes leucostictus (Müller \& Troschel, 1848) \\
\hline & & Stegastes partitus (Poey, 1868) \\
\hline
\end{tabular}


Table 1. Continued

\begin{tabular}{|c|c|c|}
\hline \multirow{2}{*}{$\begin{array}{l}\text { incertae sedis- } \\
\text { Ovalentaria } \\
\text { [continued }]\end{array}$} & \multirow{2}{*}{$\begin{array}{l}\text { Pomacentridae } \\
{[\text { continued }]}\end{array}$} & Stegastes planifrons (Cuvier, 1830) \\
\hline & & Stegastes xanthurus (Poey, 1860) \\
\hline \multirow{4}{*}{$\begin{array}{l}\text { incertae sedis- } \\
\text { Ovalentaria }\end{array}$} & \multirow{3}{*}{ Grammatidae } & Gramma loreto Poey, 1868 \\
\hline & & Gramma melacara Böhlke \& Randall, 1963 \\
\hline & & Lipogramma trilineata Randall, 1963 * \\
\hline & Opistognathidae & Opistognathus aurifrons (Jordan \& Thompson, 1905) \\
\hline Gobiesociformes & Gobiesocidae & Acyrtops beryllinus (Hildebrand \& Ginsburg, 1926) * \\
\hline \multirow{13}{*}{ Blenniiformes } & Tripterygiidae & Enneanectes boehlkei Rosenblatt, 1960 \\
\hline & \multirow{3}{*}{ Chaenopsidae } & Acanthemblemaria spinosa Metzelaar, 1919 \\
\hline & & Emblemaria pandionis Evermann \& Marsh, 1900* \\
\hline & & Hemiemblemaria simula Longley \& Hildebrand, 1940 * \\
\hline & \multirow{7}{*}{ Labrisomidae } & Gobioclinus bucciferus Poey, 1868 \\
\hline & & Malacoctenus aurolineatus Smith, 1957 * \\
\hline & & Malacoctenus boehlkei Springer, 1959 \\
\hline & & Malacoctenus macropus (Poey, 1868) \\
\hline & & Malacoctenus triangulatus Springer, 1959 \\
\hline & & Malacoctenus versicolor (Poey, 1876) \\
\hline & & Paraclinus nigripinnis (Steindachner, 1876) \\
\hline & \multirow{2}{*}{ Blenniidae } & Entomacrodus nigricans Gill, 1859 \\
\hline & & Ophioblennius macclurei (Silvester, 1915) \\
\hline \multirow{2}{*}{ Gerreiformes } & \multirow{2}{*}{ Gerreidae } & Eucinostomus lefroyi (Goode, 1874)* \\
\hline & & Gerres cinereus (Walbaum, 1792) \\
\hline \multirow{4}{*}{ Labriformes } & \multirow{4}{*}{ Labridae } & Bodianus rufus (Linnaeus, 1758) \\
\hline & & Clepticus parrae (Bloch \& Schneider, 1801) \\
\hline & & Cryptotomus roseus Cope, $1871 *$ \\
\hline & & Halichoeres bivittatus (Bloch, 1791) \\
\hline
\end{tabular}


Table 1. Continued

\begin{tabular}{|c|c|c|}
\hline ORDER & FAMILY & SPECIES \\
\hline \multirow{20}{*}{$\begin{array}{l}\text { Labriformes } \\
{[\text { continued }]}\end{array}$} & \multirow{20}{*}{$\begin{array}{l}\text { Labridae } \\
\text { [continued] }\end{array}$} & Halichoeres garnoti (Valenciennes, 1839) \\
\hline & & Halichoeres maculipinna (Müller \& Troschel, 1848) \\
\hline & & Halichoeres pictus (Poey, 1860) * \\
\hline & & Halichoeres poeyi (Steindachner, 1867) * \\
\hline & & Halichoeres radiatus (Linnaeus, 1758) \\
\hline & & Lachnolaimus maximus (Walbaum, 1792) \\
\hline & & Scarus coelestinus Valenciennes, 1840 \\
\hline & & Scarus coeruleus (Bloch, 1786) \\
\hline & & Scarus iseri (Bloch, 1789) \\
\hline & & Scarus taeniopterus Desmarest, 1801 \\
\hline & & Scarus vetula Bloch \& Schneider, 1801 \\
\hline & & Sparisoma atomarium (Poey, 1861) \\
\hline & & Sparisoma aurofrenatum (Valenciennes, 1840) \\
\hline & & Sparisoma chrysopterum (Bloch \& Schneider, 1801) \\
\hline & & Sparisoma radians (Valenciennes, 1840) \\
\hline & & Sparisoma rubripinne (Valenciennes, 1840) \\
\hline & & Sparisoma viride (Bonnaterre, 1788) \\
\hline & & Thalassoma bifasciatum (Bloch, 1791) \\
\hline & & Xyrichtys martinicensis Valenciennes, 1840 * \\
\hline & & Xyrichtys splendens Castelnau, 1855 \\
\hline \multirow{4}{*}{ Chaetodontiformes } & \multirow{4}{*}{ Chaetodontidae } & Chaetodon capistratus Linnaeus, 1758 \\
\hline & & Chaetodon ocellatus Bloch, 1787 \\
\hline & & Chaetodon striatus Linnaeus, 1758 \\
\hline & & Prognathodes aculeatus (Poey, 1860) \\
\hline $\begin{array}{l}\text { incertae sedis- } \\
\text { Eupercaria }\end{array}$ & Sciaenidae & Equetus lanceolatus (Linnaeus, 1758) \\
\hline
\end{tabular}


Table 1. Continued

\begin{tabular}{|c|c|c|}
\hline $\begin{array}{l}\text { incertae sedis- } \\
\text { Eupercaria [continued] }\end{array}$ & $\begin{array}{l}\text { Sciaenidae } \\
\text { [continued] }\end{array}$ & Equetus punctatus (Bloch \& Schneider, 1801) \\
\hline \multirow{3}{*}{ Acanthuriformes } & \multirow{3}{*}{ Acanthuridae } & Acanthurus chirurgus (Bloch, 1787) \\
\hline & & Acanthurus coeruleus Bloch \& Schneider, 1801 \\
\hline & & Acanthurus tractus Poey, 1860 \\
\hline \multirow{5}{*}{$\begin{array}{l}\text { incertae sedis- } \\
\text { Eupercaria }\end{array}$} & \multirow{4}{*}{ Pomacanthidae } & Holacanthus ciliaris (Linnaeus, 1758) \\
\hline & & Holacanthus tricolor (Bloch, 1795) \\
\hline & & Pomacanthus arcuatus (Linnaeus, 1758) \\
\hline & & Pomacanthus paru (Bloch, 1787) \\
\hline & Malacanthidae & Malacanthus plumieri (Bloch, 1786) \\
\hline \multirow{16}{*}{ Lutjaniformes } & \multirow{11}{*}{ Haemulidae } & Anisotremus virginicus (Linnaeus, 1758) \\
\hline & & Brachygenys chrysargyreum (Günther, 1859) \\
\hline & & Emmelichthyops atlanticus Schultz, 1945 \\
\hline & & Haemulon album Cuvier, 1830 \\
\hline & & Haemulon carbonarium Poey, 1860 \\
\hline & & Haemulon flavolineatum (Desmarest, 1823) \\
\hline & & Haemulon macrostomum Günther, 1859 * \\
\hline & & Haemulon parra (Desmarest, 1823) \\
\hline & & Haemulon plumierii (Lacepède, 1801) \\
\hline & & Haemulon sciurus (Shaw, 1803) \\
\hline & & Haemulon vittatum (Poey, 1860) \\
\hline & \multirow{5}{*}{ Lutjanidae } & Lutjanus analis (Cuvier, 1828) \\
\hline & & Lutjanus apodus Walbaum, 1792 \\
\hline & & Lutjanus griseus (Linnaeus, 1758) \\
\hline & & Lutjanus jocu (Bloch \& Schneider, 1801) \\
\hline & & Lutjanus mahogoni (Cuvier, 1828) \\
\hline
\end{tabular}


Table 1. Continued

\begin{tabular}{|c|c|c|}
\hline ORDER & FAMILY & SPECIES \\
\hline $\begin{array}{l}\text { Lutjaniformes } \\
\text { [continued] }\end{array}$ & $\begin{array}{l}\text { Lutjanidae } \\
\text { [continued] }\end{array}$ & Ocyurus chrysurus (Bloch, 1791) \\
\hline Lobotiformes & Lobotidae & Lobotes surinamensis (Bloch, 1790) \\
\hline Spariformes & Sparidae & Calamus calamus (Valenciennes, 1830) \\
\hline Priacanthiformes & Priacanthidae & Heteropriacanthus cruentatus (Lacepède, 1801) \\
\hline \multirow{18}{*}{ Tetraodontiformes } & \multirow{5}{*}{ Ostraciidae } & Acanthostracion polygonius Poey, 1876 \\
\hline & & Acanthostracion quadricornis (Linnaeus, 1758) \\
\hline & & Lactophrys bicaudalis (Linnaeus, 1758) \\
\hline & & Lactophrys trigonus (Linnaeus, 1758) \\
\hline & & Lactophrys triqueter (Linnaeus, 1758) \\
\hline & \multirow{2}{*}{ Diodontidae } & Diodon holocanthus Linnaeus, 1758 \\
\hline & & Diodon hystrix Linnaeus, 1758 \\
\hline & \multirow{2}{*}{ Tetraodontidae } & Canthigaster rostrata (Bloch, 1786) \\
\hline & & Sphoeroides spengleri (Bloch, 1785) \\
\hline & \multirow{5}{*}{ Monacanthidae } & Aluterus monoceros (Linnaeus, 1758) * \\
\hline & & Aluterus scriptus (Osbeck, 1765) \\
\hline & & Cantherhines macrocerus (Hollard, 1853) \\
\hline & & Cantherhines pullus (Ranzani, 1842) \\
\hline & & Monacanthus tuckeri Bean, 1906 \\
\hline & \multirow{4}{*}{ Balistidae } & Balistes capriscus Gmelin, 1789 \\
\hline & & Balistes vetula Linnaeus, 1758 \\
\hline & & Canthidermis sufflamen (Mitchill, 1815) \\
\hline & & Melichthys niger (Bloch, 1786) \\
\hline Pempheriformes & Pempheridae & Pempheris schomburgkii Müller \& Troschel, 1848 \\
\hline Centrarchiformes & Kyphosidae & Kyphosus vaigiensis (Quoy \& Gaimard, 1825) \\
\hline
\end{tabular}


Table 1. Continued

\begin{tabular}{|c|c|c|}
\hline $\begin{array}{l}\text { Centrarchiformes } \\
\text { [continued }]\end{array}$ & Cirrhitidae & Amblycirrhitus pinos (Mowbray, 1927) \\
\hline \multirow{24}{*}{ Perciformes } & \multirow{22}{*}{ Serranidae } & Cephalopholis cruentata (Lacepède, 1802) \\
\hline & & Cephalopholis fulva (Linnaeus, 1758) \\
\hline & & Epinephelus guttatus (Linnaeus, 1758) \\
\hline & & Hypoplectrus aberrans Poey, 1868 \\
\hline & & Hypoplectrus chlorurus (Cuvier, 1828) \\
\hline & & Hypoplectrus guttavarius (Poey, 1852) \\
\hline & & Hypoplectrus indigo (Poey, 1851) \\
\hline & & Hypoplectrus maculiferus (Poey, 1871)* \\
\hline & & Hypoplectrus nigricans (Poey, 1852) \\
\hline & & $\begin{array}{l}\text { Hypoplectrus providencianus Acero P. \& Garzón- } \\
\text { Ferreira, } 1994\end{array}$ \\
\hline & & Hypoplectrus puella (Cuvier, 1828) \\
\hline & & Hypoplectrus randallorum Lobel, $2011 *$ \\
\hline & & Hypoplectrus unicolor (Walbaum, 1792) \\
\hline & & Liopropoma rubre Poey, 1861 \\
\hline & & Mycteroperca bonaci (Poey, 1860) \\
\hline & & Mycteroperca interstitialis (Poey, 1860) \\
\hline & & Mycteroperca tigris (Valenciennes, 1833) \\
\hline & & Mycteroperca venenosa (Linnaeus, 1758) \\
\hline & & Rypticus saponaceus (Bloch \& Schneider, 1801) \\
\hline & & Serranus baldwini (Evermann \& Marsh, 1899) * \\
\hline & & Serranus tabacarius (Cuvier, 1829) \\
\hline & & Serranus tigrinus (Bloch, 1790) \\
\hline & \multirow{2}{*}{ Scorpaenidae } & Pterois volitans (Linnaeus, 1758) \\
\hline & & Scorpaena plumieri Bloch, 1789 \\
\hline
\end{tabular}

$\S$ Name favored for the Southern Sennet following van der Laan and Fricke (2020). 


\section{ACKNOWLEDGMENTS}

We want to extend special recognition to the Comisión Colombiana del Océano (CCO) for organizing and leading the expedition to Cayo Albuquerque. Contribution 508 of Cecimar. Diego Cardeñosa (Colombia Azul Foundation), Gina Clementi (Florida International University), Ryan Logan (NOVA Southern University), Mateo López (Pontificia Universidad Javeriana Cali), Néstor Hernando Campos (Cecimar, Universidad Nacional de Colombia), and Alexandra Pineda (Cecimar, Universidad Nacional de Colombia) contributed with data and field information. Santiago Estrada (Reef Shepherd Dive Center) shared his photographic records. Two anonymous reviewers contributed many improvements to the manuscript.

\section{REFERENCES}

Abril-Howard, A., N. Bolaños, I. Machacón, J. Lasso, D. I. Gómez, and V. Ward. 2012. Actualización del conocimiento de los ecosistemas marinos en la Reserva de la Biosfera Seaflower, con énfasis en las islas de San Andrés y Providencia. In Atlas de la Reserva de Biósfera Seaflower. Archipiélago de San Andrés, Providencia y Santa Catalina, ed. D. I. Gómez-López, C. Segura-Quintero, P. C. Sierra-Correa, and J. Garay-Tinoco, pp. 129-157. Santa Marta, Colombia: Instituto de Investigaciones Marinas y Costeras.

Acero P., A., J. J. Tavera, A. Polanco F., and N. Bolaños-Cubillos. Fish biodiversity in three northern islands of the Seaflower Biosphere Reserve, Colombian Caribbean. Frontiers in Marine Science 6 (113): 1-11. https://doi.org/10.3389/fmars.2019.00113

Betancur-R., R., E. O. Wiley, G. Arratia, A. Acero P., N. Bailly, M. Miya, G. Lecointre, and G. Ortí. 2017. Phylogenetic classification of bony fishes. BMC Evolutionary Biology 17(162). https://doi.org/10.1186/s12862-017-0958-3

Birdsong, R. S., and A. R. Emery. 1967. New records of fishes from the western Caribbean. Quarterly Journal of the Florida Academy of Sciences 30(3): 187-196.

Bolaños-Cubillos, N., A. Abril-Howard, H. Bent-Hooker, J. P. Caldas, and A. Acero P. 2015. Lista de peces conocidos del archipiélago de San Andrés, Providencia y Santa Catalina, Reserva de Biosfera Seaflower, Caribe. Boletín de Investigaciones Marinas y Costeras 44(1): 127-162. https://doi.org/10.25268/bimc.invemar.2015.44.1.24

Chasqui, V. L., A. Polanco F., A. Acero P., P. A. Mejía-Falla, A. Navia, and L. Zapata., eds. 2017. Libro rojo de peces marinos de Colombia. Serie Publicaciones Generales Invemar 93, Santa Marta, Colombia.

Fowler, H. W. 1944. The fishes. In Results of the Fifth Vanderbilt Expedition (1941), Monographs of the Academy of Natural Sciences of Philadelpha No. 6, pp. 57-529. Philadelphia: Academy of Natural Sciences of Philadelphia.

Gamboa, L., O. B. Posada, O. C. González, G. Hurtado, and C. A. Andrade-Amaya. 2012. Descripción física del Archipiélago de San Andrés, Providencia y Santa Catalina. In Atlas de la Reserva de Biósfera Seaflower. Archipiélago de San Andrés, Providencia y Santa Catalina, ed. D. I. Gómez-López, C. Segura-Quintero, P. C. Sierra-Correa, and J. Garay-Tinoco, pp. 35-53. Santa Marta, Colombia: Instituto de Investigaciones Marinas y Costeras.

Gómez, D. P., and P. Victoria. 1986. Peces de la isla de San Andrés y noreste de la isla de Providencia (mar Caribe de Colombia): inventario en arrecifes coralinos, praderas marinas y aguas costeras. Boletín Ecotrópica 13: 41-85.

Mejía, L. S., J. Garzón-Ferreira, and A. Acero P. 1998. Peces registrados en los complejos arrecifales de los cayos Courtown, Albuquerque y los bancos Serrana y Roncador, Caribe occidental, Colombia. Boletín Ecotrópica 32: 25-42. 
Nelson, J. S., T. C. Grande, and V. H. Wilson. 2016. Fishes of the World. Hoboken, N.J.: John Wiley \& Sons, Inc.

Robertson, D. R., E. A. Peña, J. M. Posada, and R. Claro. 2019. Peces costeros del Gran Caribe: sistema de información en línea. Version 2.0. Smithsonian Tropical Research Institute, Balboa, Panama.

Taylor, E., M. Howard, R. Medina, and O. Bent. 2012. Gestión ambiental de la Reserva de la Biosfera Seaflower, archipiélago de San Andrés, Providencia y Santa Catalina, Caribe occidental, Colombia. In Atlas de la Reserva de Biósfera Seaflower. Archipiélago de San Andrés, Providencia y Santa Catalina, ed. D. I. Gómez-López, C. Segura-Quintero, P. C. Sierra-Correa, and J. Garay-Tinoco, pp. 13-33. Santa Marta, Colombia: Instituto de Investigaciones Marinas y Costeras.

Van der Laan, R., and R. Fricke. 2020. Eschmeyer's catalog of fishes: family-group names. https://www.calacademy.org/scientists/catalog-of-fishes-family-group-names (accessed 23 October 2020).

Victoria, P., and D. P. Gómez. 1984. Nuevos registros de peces para la isla de San Andrés (mar Caribe de Colombia). Anales del Instituto de Investigaciones Marinas de Punta Betín 14: 115-132. 